\title{
The Targeted Management (TEAM) Intervention for Reducing Stroke Risk in African American Men: Rationale and Study Design of a Prospective Randomized Controlled Trial
}

This article was published in the following Dove Press journal:

Journal of Multidisciplinary Healthcare

\author{
Carolyn H Still $\mathbb{D}^{\prime}$ \\ Chris Burant' \\ Shirley Moore' \\ Doug Einstadter 2,3 \\ Cheryl Killion' \\ Charles Modlin ${ }^{4}$ \\ Sophia Sundararajan ${ }^{5,6}$ \\ John D Thornton 3,7 \\ Jackson T Wright Jr ${ }^{5,6}$ \\ Martha Sajatovic ${ }^{6,8}$ \\ 'Frances Payne Bolton, School of \\ Nursing, Case Western Reserve \\ University, Cleveland, OH, USA; ${ }^{2}$ Center \\ for Health Care Research and Policy, \\ Case Western Reserve University, \\ Cleveland, OH, USA; ${ }^{3}$ The MetroHealth \\ System, Cleveland, OH, USA; ${ }^{4}$ Glickman \\ Urological and Kidney Institute, \\ Cleveland Clinic, Cleveland, OH, USA; \\ ${ }^{5}$ Case Western Reserve University, \\ School of Medicine, Cleveland, OH, USA; \\ ${ }^{6}$ University Hospitals Cleveland Medical \\ Center, Cleveland, OH, USA; ${ }^{7}$ Center for \\ Reducing Health Disparities, Case \\ Western Reserve University, Cleveland, \\ $\mathrm{OH}$, USA; ${ }^{8}$ Department of Psychiatry and \\ of Neurology, Case Western Reserve \\ University, School of Medicine, Cleveland, \\ $\mathrm{OH}$, USA
}

Correspondence: Carolyn H Still

Frances Payne Bolton, School of Nursing, Case Western Reserve University, 10900 Euclid Ave, Cleveland, OH, 44I06, USA

Tel: + I 2163686338

Email cwhII@case.edu
Background: African American (AA) male survivors of strokes or transient ischemic attacks (TIA) have the highest risk of recurrent stroke when compared to other racial-ethnic men. However, there is a paucity of evidence-based strategies, including organizational, educational, or behavioral interventions, that targets secondary stroke risk reduction in AA men.

Methods: Targeted Management for Reducing Stroke Risk (TEAM) is an ongoing, 6-month prospective, randomized controlled trial that will determine whether a curriculum-guided self-management approach, using peer dyads (men who had a stroke or TIA and their care partners) will improve post-stroke care in AA men.

Results: The study sample will consist of 160 AA men who have experienced a stroke or TIA within 5 years, randomized to TEAM or Wait-list control group. The primary outcome changes in systolic blood pressure (BP) and high-density lipoprotein (HDL), while secondary outcomes include diastolic BP, total cholesterol, low-density lipoprotein, triglycerides, and glycemic control for diabetics. We hypothesize that AA men in TEAM will have significantly lower systolic BP and higher HDL when compared to AA men in the Wait-list control group at 6-month.

Conclusion: Persistent disparities for stroke burden in AA men highlight the need for novel interventions to promote secondary stroke-risk reduction. Building on promising pilot data, TEAM uses a group format, with a nurse and patient co-led intervention focused on AA men and family needs, practice in problem-solving, and attention to emotional and role management. In addition, the TEAM approach may help reduce stroke risk factors and health disparities in AA men.

Clinicaltrials.gov Identifier: NCT04402125.

Keywords: stroke, transient ischemic attack, stroke prevention, African-Americans, health disparities

\section{Introduction}

Stroke is the fifth leading cause of death in the United States. ${ }^{1}$ While stroke mortality has declined over the last decade, African Americans (AA) are twice as likely to suffer from a stroke at a younger age $(<44$ years old $)$ and have higher mortality rates when compared to other racial-ethnic groups. ${ }^{1,2}$ In 2016, nearly 800,000 Americans had a stroke, costing the US healthcare system \$34 billion dollars. ${ }^{2,3}$ National vital statistics show the age-adjusted death rate for stroke is 
markedly higher in AA men when compared to the overall US population (56.7 versus 37.3 per 100,000), including women. $^{2,3}$

AA stroke mortality and incidence are partially explained by disparities in their stroke risk profiles and are particularly high among AA men. ${ }^{4,5}$ Racial differences in prevalence, awareness, treatment, and control of stroke risk factors, such as hypertension, hyperlipidemia, obesity, and diabetes largely contribute to the higher adverse events and stroke incidence in AA men. ${ }^{2,6-8}$ Evidence also suggests that social determinants of health, stress, and cultural factors all play a role in stroke disparities and health outcomes. ${ }^{4,8,9}$ Moreover, race-specific inequalities (eg, discrimination, stigmatization, and microaggression) trigger individual pathophysiological changes to the body that contribute to the disproportionate burden of stroke in AA men. ${ }^{10,11}$ These problems subsequently result in stress or the inability to cope with adversity and lead individuals to adopt unhealthy behaviors such as smoking, poor nutrition, or physical inactivity. Notably, all of these factors can potentially increase the risk of a mini-stroke (transient ischemic attack [TIA]), a precursor to a stroke, occurring before $12 \%$ of all strokes. ${ }^{1,2}$

More importantly, not only is the burden of first-time stroke higher in AA, the risk of recurrent stroke is higher in AA when compared to non-AA. ${ }^{12}$ Recurrent stroke risk increases over time and $25 \%$ of stroke survivors will die of a recurrent stroke. ${ }^{13,14} \mathrm{~A}$ recent analysis of a global, multicenter clinical trial focused on stroke and cardiovascular event recurrence evaluated 3,470 (20\% AA) recent stroke patients (aged 35 years or older) and followed them for 2 years. ${ }^{15}$ AA patients (enrolled in the US and United Kingdom) with a recent stroke had approximately $60 \%$ higher risk of recurrent stroke compared to their white counterparts. ${ }^{15}$ This difference was explained by a substantially greater vascular risk factor burden in the AA. ${ }^{15}$ Furthermore, these results suggest that targeted care for better risk factor control after a stroke in AA may help close the disparity gap in racial stroke outcomes. ${ }^{16}$

\section{Prevention of Secondary Stroke to Improve Post-Stroke Outcomes}

Evidence is growing regarding the contribution and the interplay of modifiable risk factors and the reduction of stroke mortality. ${ }^{17} \mathrm{~A}$ statement by the American Heart Association/American Stroke Association (AHA/ASA) on the prevention of incident or recurrent stroke highlights interventions to improve post-stroke outcomes and reduce the risk for future stroke. ${ }^{17}$ These interventions include evidence-based pharmacotherapies ${ }^{18}$ and aggressive management of stroke risk factors, such as hypertension, ${ }^{19}$ hyperlipidemia, ${ }^{20}$ and diabetes. ${ }^{21}$ In addition, recommendations for behavioral modifications, such as smoking cessation, change in diet, weight loss, exercise, and stress management, are strategies shown to reduce secondary stroke mortality. ${ }^{17}$ However, AA men have the greatest underuse of evidence-based post-stroke care for multifactorial reasons, including low socioeconomic status, inadequate health insurance, and poor access or referral to outpatient rehabilitation. ${ }^{4,22}$ Unfortunately, this is compounded by low AA stroke risk factor awareness that might prevent future stroke. ${ }^{23}$ These persistent challenges highlight the need for further efforts to reduce the risk of recurrent stroke in AA. ${ }^{24-28}$

To improve post-stroke care, self-management interventions are recommended as a method of supporting individuals to change behavior and take an active part in managing their own health, ${ }^{21,29}$ as well as develop skills to monitor, cope, and adapt to living poststroke. ${ }^{30,31}$ While there are a number of reports on selfmanagement for stroke survivors, ${ }^{16,31-34}$ there is a paucity of evidence-based strategies, including organizational, educational, or behavioral interventions that target secondary stroke risk reduction in AA men. ${ }^{35}$ The Prevent Recurrence of All Inner-city Strokes through Education (PRAISE) trial developed a culturally tailored, peer-led, and community-based chronic disease self-management program to address reducing stroke risk factors among minority stroke survivors. ${ }^{33}$ Results suggest that the PRAISE was associated with a reduction in blood pressure (BP) in individuals who had experienced stroke or TIA within the past 5 years. ${ }^{34}$ However, the study was comprised of mostly females $(60 \%)$ and did not demonstrate significant improvements in other health indicators (eg, lowdensity lipoprotein [LDL], use of antithrombic medication). Importantly, among factors contributing to stroke risk disparities in AA, systolic BP is a critical modifiable risk factor that accounts for half of the combined Framingham risk factor effect. ${ }^{8}$ Thus, approaches that target systolic BP in AA men and other health indicators (Lipids, cholesterol, hemoglobin A1C) may help reduce stroke risk factors and have the potential to close the stroke disparity gap in AA men. 


\section{Integrating Targeted Management for Stroke Risk Reduction}

Targeted Management (TEAM) is a novel intervention, developed by this study team, ${ }^{36}$ to promote secondary stroke-risk reduction in AA men at high-risk for recurrent stroke. TEAM uses a group format, with a nurse and patient co-led intervention focused on patient and family needs, practice in problem-solving, and attention to emotional and role management in stroke risk reduction. Preliminary work testing the feasibility, acceptability, and efficacy of TEAM (versus treatment as usual [TAU]) in AA men within 1-year of experiencing a stroke or TIA demonstrated improvements in mean systolic BP, high-density lipoprotein cholesterol (HDL), and glycosylated hemoglobin ( $\mathrm{HbA1c}$ ) at 24 weeks among AA men receiving the TEAM intervention.

TEAM aligns with the Individual and Self-Management Theory (ISMT) of Ryan and Sawin ${ }^{37}$ and combines selfmanagement training, peer support and behavioral modeling within a family framework that emphasizes the involvement and engagement of the family, acknowledges the presence and variability of family/support network stress, and is culturally sensitive to the needs of AA men. Given the lack of post-stroke risk reduction approaches specifically targeting AA men, TEAM has the potential to advance care and reduce stroke risk in this vulnerable population.

\section{Purpose}

This paper describes the design and methods of a 6-month prospective, randomized controlled trial (RCT) to examine the effects of TEAM versus a Wait-list control group on systolic BP and HDL in AA men with stroke or TIA. We hypothesize that AA men in TEAM will have significantly lower systolic BP and higher HDL levels when compared to AA men in the Wait-list control group at 6-month follow-up.

\section{Methods and Materials}

\section{Study Design}

The proposed project is a 6-month prospective RCT comparing the TEAM intervention and Wait-list control group (Figure 1). This research protocol was approved by University Hospitals Cleveland Medical Center's (UHCMC) Institutional Review Board and will be conducted in accordance with this trial will be conducted in accordance with the Declaration of Helsinki. All study participants will provide written informed consent prior to study enrollment.

To optimize study enrollment and retention as well as collect information on longer-term trajectories of outcomes, individuals randomized to TEAM will be followed for a total of 12 months; baseline to 6 months for the initial RCT evaluation and from 6 to 12 months post-baseline to evaluate the sustainability of the TEAM intervention. Individuals in the Wait-list control group will receive the TEAM intervention for a 6-month period after a delay, starting at month 6 and concluding at month 12 (postbaseline). The primary health outcome (Aim 1) is a change in systolic BP, while secondary health outcomes (Aim 2) include a change in other biologic parameters of stroke risk-diastolic BP, low-density lipoprotein (LDL), HDL, triglycerides, and glycemic control (HbAlc) for diabetics. Other goals of this study include exploring how contextual factors (age, individual and neighborhood stress, racial discrimination, perceived health, depression, anxiety, social role, health literacy, CVD-related factors), process factors (stroke knowledge, self-efficacy, perceived social support), and proximal health behaviors (diet, exercise, smoking, and tobacco/substance use) may impact the primary and secondary health outcomes. Qualitative assessment will evaluate TEAM and Wait-list control group from both the perspective of AA men and their care partners (someone who is involved in their stroke recovery care or support network).

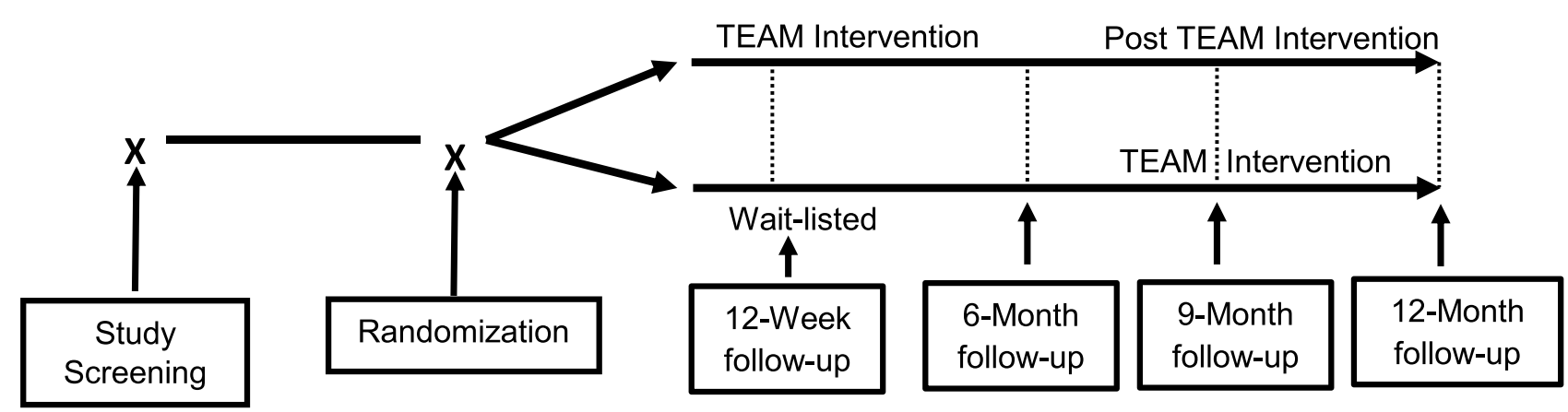

Figure I Randomized control trial study design. 


\section{Participants}

The study sample will consist of approximately 160 AA men who have experienced a stroke or TIA and are within 5 years of a hospital discharge from an acute stroke program or Emergency Department visit for TIA. TIA categorization will be based upon the ABCD TIA score, ${ }^{38-40}$ a statistically and clinically valid method of identifying individuals with TIA. Eligible participants will be enrolled if they meet the following inclusion criteria: 1) selfidentified as AA male, 2) current Barthel Index (BI) score of $>60$ (indicating no more than mild-moderate stroke-related functional deficit), ${ }^{41,42}$ and 3) able to participate in groups. Participants will be excluded if they are unwilling or unable to provide informed consent or if their stroke was felt to be related to sickle-cell disease. Involvement of care partners will be encouraged but not a requirement for this study.

The sample size for this study was determined based on the authors' previous pilot work, ${ }^{36}$ which demonstrated significant group differences on the primary outcome systolic BP at 6 months $(p=0.03)$. Moreover, power analysis was calculated for RMANOVA, testing between-group differences (TEAM and Wait-list control) would achieve adequate power with a sample size of 38 for each group. Allowing for a conservative $30 \%$ dropout, using an alpha of 0.05 , a moderate to small effect size of 0.21 , and power of 0.80 indicates a final sample of $160(n=80$ in each group) will be sufficient to detect differences between the study groups.

\section{Setting}

This study will take place in Cleveland, $\mathrm{OH}-\mathrm{a}$ large metropolitan city in the Midwest, where the population is approximately 384,000 people, the median age is 36 , and the median household income is $\$ 28,000$. AA makes up $50 \%$ of the study site population and $25 \%$ live below the federal poverty level. ${ }^{43}$

\section{Recruitment}

Participants will be recruited from two large academic medical centers in Northeast Ohio. Methods for identifying potentially eligible participants within the clinical practice of the research settings will include a targeted review of medical records or databases for those meeting the trial's inclusion criteria, Institutional Review Board (IRB)-approved posted advertisements, direct mailings, and referrals from providers/employees within the practice and/or from practice participants themselves.

\section{Treatment Randomization}

After informed consent, and screening and baseline procedures, individuals will be randomized on a $1: 1$ basis to either TEAM $(n=80)$ or Wait-listed control $(n=80)$. Block randomization with block sizes ranging randomly between 4 and 8 consecutive patients will be employed to ensure that equal numbers of TEAM and Wait-list control participants occur within strata and are balanced with respect to relevant comorbidities (diabetes and previous stroke). The randomization list will be computer-generated by biostatisticians who are not members of the study staff.

\section{Intervention}

\section{TEAM Intervention Arm}

We have developed a curriculum-guided selfmanagement approach that promotes self-management training, peer support, and behavioral modeling to reduce stroke risk in AA men post-stroke/TIA. ${ }^{6,44}$ The TEAM intervention (Figure 2) consists of individual and group sessions, as well as telephone follow-up sessions. TEAM sessions are delivered by a nurse educator and a peer dyad. The Peer Dyad (or PED) consists of an AA male who has experience in managing his own stroke risk and his care partner. The steps of the interventions are described next.

First, there is a single individual session with the participant, his care partner (if applicable), the nurse educator and a PED. The initial session covers introductions, orientation, and logistic planning and takes place within 30 days of study enrollment and prior to the first TEAM group session. Next, participants will complete five interactive group sessions. Each group session will include 6-10 AA men with their care partner and occur every 2 weeks beginning at 4 weeks. Sessions are co-led by a nurse and a PED and will last approximately 60 minutes. Following the group-session series, participants in TEAM will have six telephone maintenance sessions, spaced approximately every 2 weeks with the nurse educators or a member of the PED in an alternating fashion. Phone sessions will reinforce content from the group sessions, serve as a behavioral mode, and provide social support.

\section{Wait-List Control Arm}

Individuals randomized to the Wait-list control group will participate in their regular medical care for 6 months. 


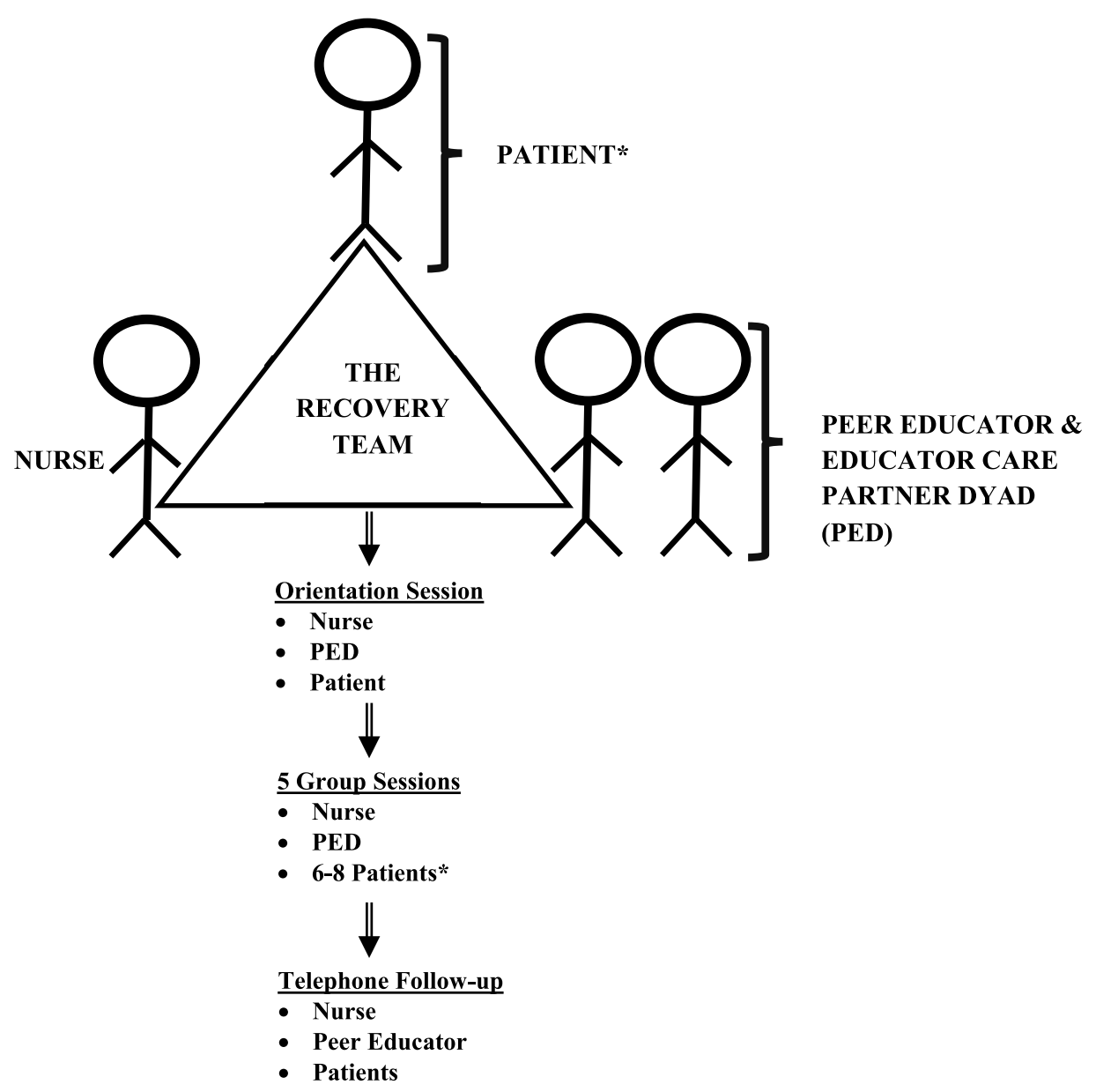

Figure 2 TEAM participants and encounters. *Patient care partner also encouraged to participate if available.

After 6 months, the Wait-list group will receive the TEAM intervention as a supplement to their regular medical care for 6 months.

\section{Procedures}

After screening and enrollment, each research participant (in both groups) will be assessed at baseline, 3, 6, 9, and 12 months. Data will be obtained from patient interviews, BP evaluation, and laboratory testing (collected at baseline, 6 and 12 months). Laboratory testing will be conducted only will be collected by registered nurses on the clinical research unit. Trained research assistants will conduct study visits, while registered nurses on a clinical research unit will collect BP and laboratory data. Fidelity to TEAM will be evaluated by non-interventionist study staff randomly attending $20 \%$ of sessions using a standardized check-list to determine if sessions covered relevant TEAM processes, content, and format as appropriate.

\section{Measures}

Baseline assessment will include self-reported demographic information as well as contextual, process, behavioral and health outcome factors relevant to the ISMT theoretical model. Study measures are described below. All data will be collected and managed in electronic data, REDCap (Research Electronic Data Capture).

\section{Primary and Secondary Health Outcomes}

The primary health outcome is a change in systolic BP from baseline to 6-month follow-up between TEAM vs Wait-list control participants. BP assessments will be conducted using standardized accepted procedures (appropriate cuff size, proper positioning, after 5 minutes of rest), ${ }^{45,46}$ and based on the current 2017 American College of Cardiology/American Heart Association (ACC/AHA) blood pressure guidelines. ${ }^{19}$ Additional biological markers indicative of stroke risk will be collected, including diastolic BP, body mass index (BMI), and 
blood specimens (total cholesterol, HDL, LDL, triglycerides, $\mathrm{HbAlc}$ ).

\section{Contextual Factors}

Variables known to contribute to racial health disparities as well as individual and health condition-specific factors that may impact the effects of TEAM on AA men were considered. Individual perceived stress will be evaluated with Cohen's Perceived Stress Scale (PSS; $\alpha=0.78),{ }^{47,48}$ a 10-item global health instrument that has been validated in the USA Neighborhood stress will be characterized using the residential neighborhood codes used by the regional Northeast Ohio Community and Neighborhood Data for Organizing (NEOCANDO) project (http://neocando.case.edu). The Patient Rated Outcomes Measurement Information System (PROMIS; $\alpha=0.91$ to 0.98 ) is a 29 -item comprehensive selfrated measure that will be used to evaluate the perceived health status of one's physical function, anxiety, depression, social role, sleep, fatigue and pain. ${ }^{49,50}$ Perceived racial discrimination will be assessed with the 9-item Everyday Discrimination Scale (EDS) developed by Williams and colleagues. ${ }^{51}$ A recent systematic literature review on studies that evaluated the links between racial discrimination and hypertension noted that the EDS was one of the most widely used instruments on this topic. ${ }^{52}$ Health literacy will be measured by the Rapid Estimate of Adult Literacy in Medicine short form $(\alpha=$ 0.95). ${ }^{53}$ Physical health contextual factors will include medical burden ${ }^{18,54}$ measured by the Cardiovascular Health Index. ${ }^{55}$ To evaluate the patient/care partner relationship the Mutuality Scale, a 15 -item instrument, will be used to assess their relationship in terms of love, shared pleasurable activities, common values, and reciprocity. ${ }^{56}$

\section{Self-Management Processes}

Stroke knowledge will be measured with the Stroke Knowledge Test $(\alpha=0.65),{ }^{57,58}$ to examine knowledge of keystroke risk factors and stroke prevention practices. Self-efficacy will be measured with the General SelfEfficacy Scale $(\alpha=0.76-0.90) .{ }^{59,60}$ Social support will be measured with the Medical Outcomes Study Social Support Survey $(\alpha=0.91){ }^{61,62}$

\section{Proximal Behavioral Outcomes}

Health behaviors known to be important to stroke risk reduction-adherence with medication, healthy diet, exercise and avoidance of alcohol and recreational drugs will be assessed. Medication-taking will be assessed in two ways: 1) whether individuals are taking indicated stroke risk-reduction drugs (anticoagulants, antiplatelet agents, antihypertensive drugs, lipid-lowering drugs and drugs for diabetes); and 2) self-reported medication adherence using the Tablets Routine Questionnaire. ${ }^{58}$ The Nutrition Data System for Research will be used to evaluate diet within the past 3 days. ${ }^{63,64}$ Exercise will be assessed with the Morgenstern Physical Activity Questionnaire $(\alpha=0.87){ }^{65}$ Smoking will be assessed with the Fagerström Test for Nicotine Dependence, ${ }^{66,67}$ while the use of alcohol and other recreational drugs will be assessed with the National Institute on Drug Abuse-Modified Alcohol, Smoking, and Substance Involvement Screening $(\alpha=0.77)^{68,69}$ for adults.

\section{Qualitative Interviews}

In-depth interviews will be conducted by trained research assisted with 40 patients at baseline (half from TEAM and half from Wait-list control) to explore the perceptions of AA men post-stroke/TIA regarding barriers/facilitators, coping with stress and chronic illness, and self-managing modifiable risk factors. Individuals who provide baseline data will be interviewed again at 6-months to further understand how they are managing their stroke risk factors and what has helped them over the past 6 months in stroke recovery. In addition, interviews will be conducted with 20 care partners (10 from TEAM and 10 from Wait-list control) at baseline and 6-month follow-up to determine what they felt were the most important and helpful factors in managing stroke risk and how it might potentially be improved in the future.

\section{Analyses}

Preliminary data analysis will include examining the frequencies of items to identify the range of variability of each item study measure. Descriptive statistics will include examining means, standard deviations, and testing for normality using skewness and kurtosis. The primary analysis will compare mean differences in systolic BP across groups (TEAM vs Wait-list control) over the initial 6 months of the study. Analyses will also compare followup from 6 to 12 months to assess the sustainability of the TEAM intervention using repeated-measures analysis of variance (RMANOVA).

We will examine the extent to which the selfmanagement process and behavioral outcomes mediate how the TEAM intervention impacts primary and secondary outcomes (systolic BP, diastolic BP, HDL, serum lipids, HbA1c) over 12 months. In the proposed study, we intend to use an autoregressive (AR) contemporaneous model to gain a better understanding of how the selfmanagement process impacts behavioral outcomes, which 
in turn allows us to test how behavioral outcomes impact primary and secondary outcomes across five time waves over a year.

\section{Discussion}

As AA men underutilize evidence-based post-stroke care, there is a critical need for interventions that maximize facilitators and minimize barriers to care. Interventions that improve post-stroke care for AA men and reduce stroke risk factors have the potential to close the health disparity gap between AA men and other groups. This paper describes the design and methods of an ongoing study for reducing stroke risk in AA men.

This study builds upon promising pilot work which developed and tested a curriculum-guided self-management approach-TEAM Intervention to specifically target and reduce stroke risk factors in AA men using AA peer educators (patients who have had stroke or TIA) as part of the intervention. Data from a 6-month prospective pilot randomized controlled trial conducted by this team comparing TEAM ( $n=19)$ vs treatment as usual (TAU, $n=19)$ found that TEAM participants had significantly lower systolic BP after 6 months $(p=0.03){ }^{6,36}$ Thus, this self-management intervention, targeted to minority men at high risk for stroke may reduce recurrent stroke burden in AA men.

This study is innovative in various ways: 1) it focuses on AA males, 2) it uses peer educator dyads (PED) composed of an AA male peer educator and his care partner to help deliver the intervention in collaboration with a nurse, 3) it includes other support system individual as both educators and as recipients of support/education, 4) it uses a curriculum-driven self-management program, which has rarely been used in studies of AA men, and 5) the investigation was informed by ISMT framework, ${ }^{37}$ a conceptual model that may help explain salient experimental elements. Taken together, the health disparities focus, use of AA men as both teachers and learners, ISMT conceptual framework, and detailed curriculum represent a new research direction with the potential to advance care for AA men to reduce stroke risk.

The information gained from this study may improve the outcomes of AA men post a stroke or TIA. Treatment and selfmanagement among individuals who have experienced these conditions is a problem of critical public health importance. The proposed research features a person-centered, holistic intervention that takes advantage of existing strengths in AA families and communities, including peer dyads who have experienced the burden of stroke. The intervention represents a practical and generalizable approach suitable for implementation in specialty, primary care or community settings, and has the potential to reverse the unacceptably high morbidity seen in AA men due to stroke and stroke-related disorders.

\section{Acknowledgments}

This research is supported by the National Institute of Nursing Research of the National Institutes of Health (1R01NR018023-01A1) and the Clinical and Translational Science Collaborative of Cleveland, UL1TR000439 from the National Center for Advancing Translational Sciences (NCATS) component of the National Institutes of Health and NIH Roadmap for Medical Research. The content is solely the responsibility of the authors and does not necessarily represent the official views of the National Institutes of Health.

\section{Author Contributions}

All authors made substantial contributions to conception and design, acquisition of data, or analysis and interpretation of data; took part in drafting the article or revising it critically for important intellectual content; agreed to submit to the current journal; gave final approval of the version to be published; and agree to be accountable for all aspects of the work.

\section{Disclosure}

Dr Martha Sajatovic reports grants from the National Institute of Nursing Research to institution, during the conduct of the study; grants from Nuromate, Otsuka, Alkermes, International Society for Bipolar Disorders (ISBD), National Institutes of Health (NIH), Centers for Disease Control and Prevention (CDC), and Patient-Centered Outcomes Research Institute (PCORI); personal fees from Alkermes, Otsuka, Janssen, Neurocrine, Bracket, Health Analytics, and Frontline Medical Communications; publication royalties from Springer Press, Johns Hopkins University Press, Oxford Press, and UpToDate; compensation for the preparation of continuing medical education materials from American Physician's Institute, MCM Education, CMEology, Potomac Center for Medical Education, Global Medical Education, Creative Educational Concepts, and Psychopharmacology Institute, outside the submitted work. The authors have no other conflicts of interest.

\section{References}

1. Virani SS, Alonso A, Benjamin EJ, et al. Heart disease and stroke statistics-2020 update: a report from the American Heart Association. Circulation. 2020;141(9):e139-e596. doi:10.1161/ CIR.0000000000000757 
2. American Heart Association. 2019 health disease \& stroke statistical update fact sheet blacks \& cardiovascular diseases. 2019. Available from: https://professional.heart.org/en/science-news/heart-diseaseand-stroke-statistics-2019-update. Accessed October 1, 2020.

3. Centers for Disease Control and Prevention. Stroke fact sheet. 2018. Available from: https://www.cdc.gov/stroke/facts.htm. Accessed November 1, 2019.

4. Cruz-Flores S, Rabinstein A, Biller J, et al. Racial-ethnic disparities in stroke care: the American experience: a statement for healthcare professionals from the American Heart Association/American Stroke Association. Stroke. 2011;42(7):2091-2116. doi:10.1161/ STR.0b013e3182213e24

5. Davis SK, Gebreab S, Quarells R, Gibbons GH. Social determinants of cardiovascular health among black and white women residing in Stroke Belt and Buckle regions of the South. Ethn Dis. 2014;24 (2):133-143.

6. Blixen C, Perzynski A, Cage J, et al. Stroke recovery and prevention barriers among young african-american men: potential avenues to reduce health disparities. Top Stroke Rehabil. 2014;21(5):432-442. doi: $10.1310 /$ tsr2105-432

7. Gutierrez J, Williams OA. A decade of racial and ethnic stroke disparities in the United States. Neurology. 2014;82(12):1080-1082. doi:10.1212/WNL.0000000000000237

8. Howard VJ. Reasons underlying racial differences in stroke incidence and mortality. Stroke. 2013;44(6 Suppl 1):S126-128. doi:10.1161/ STROKEAHA.111.000691

9. Ostwald SK, Bernal MP, Cron SG, Godwin KM. Stress experienced by stroke survivors and spousal caregivers during the first year after discharge from inpatient rehabilitation. Top Stroke Rehabil. 2009;16 (2):93-104. doi:10.1310/tsr1602-93

10. Hall JM, Fields B. "It's Killing Us!" narratives of black adults about microaggression experiences and related health stress. Glob Qual Nurs Res. 2015;2:2333393615591569. doi:10.1177/2333393615591569

11. Perry BL, Harp KL, Oser CB. Racial and gender discrimination in the stress process: implications for African American Women's Health and well-being. Sociol Perspect. 2013;56(1):25-48. doi:10.1525/ sop.2012.56.1.25

12. Albright KC, Huang L, Blackburn J, et al. Racial differences in recurrent ischemic stroke risk and recurrent stroke case fatality. Neurology. 2018;91(19):e1741-e1750. doi:10.1212/WNL.0000 000000006467

13. Hardie K, Hankey GJ, Jamrozik K, Broadhurst RJ, Anderson C. Tenyear survival after first-ever stroke in the Perth community stroke study. Stroke. 2003;34(8):1842-1846. doi:10.1161/01.STR.000 0082382.42061.EE

14. Whisnant JP, Wiebers DO, O'Fallon WM, Sicks JD, Frye RL. Effect of time since onset of risk factors on the occurrence of ischemic stroke. Neurology. 2002;58(5):787-794. doi:10.1212/WNL.58.5.787

15. Park JH, Ovbiagele B. Association of black race with recurrent stroke risk. J Neurol Sci. 2016;365:203-206. doi:10.1016/j.jns.2016.04.012

16. Bridgwood B, Lager KE, Mistri AK, Khunti K, Wilson AD, Modi P. Interventions for improving modifiable risk factor control in the secondary prevention of stroke. Cochrane Database Syst Rev. 2018;5:Cd009103. doi:10.1002/14651858.CD009103.pub3

17. Kernan WN, Ovbiagele B, Black HR, et al. Guidelines for the prevention of stroke in patients with stroke and transient ischemic attack: a guideline for healthcare professionals from the American Heart Association/ American Stroke Association. Stroke. 2014;45(7):2160-2236.

18. Linn BS, Linn MW, Gurel L. Cumulative illness rating scale. J Am Geriatr Soc. 1968;16(5):622-626. doi:10.1111/j.1532-5415.1968.tb02103.x

19. Whelton PK, Carey RM, Aronow WS, et al. 2017 ACC/AHA/AAPA/ $\mathrm{ABC} / \mathrm{ACPM} / \mathrm{AGS} / \mathrm{APhA} / \mathrm{ASH} / \mathrm{ASPC} / \mathrm{NMA} / \mathrm{PCNA}$ guideline for the prevention, detection, evaluation, and management of high blood pressure in adults a report of the American College of Cardiology/ American Heart Association task force on clinical practice guidelines. Hypertension. 2017.
20. Grundy SM, Stone NJ, Bailey AL, et al. 2018 AHA/ACC/AACVPR/ AAPA/ABC/ACPM/ADA/AGS/APhA/ASPC/NLA/PCNA Guideline on the management of blood cholesterol: a report of the American College of Cardiology/American Heart Association task force on clinical practice guidelines. J Am Coll Cardiol. 2019;73(24):e285e350.

21. Arnett DK, Blumenthal RS, Albert MA, et al. 2019 ACC/AHA guideline on the primary prevention of cardiovascular disease: executive summary: a report of the American College of Cardiology/ American Heart Association task force on clinical practice guidelines. J Am Coll Cardiol. 2019;74(10):1376-1414.

22. Carnethon MR, Pu J, Howard G, et al. Cardiovascular health in African Americans: a scientific statement from the American Heart Association. Circulation. 2017;136(21):e393-e423. doi:10.1161/ CIR.0000000000000534

23. Howard G, Prineas R, Moy C, et al. Racial and geographic differences in awareness, treatment, and control of hypertension: the reasons for geographic and racial differences in stroke study. Stroke. 2006;37(5):1171-1178. doi:10.1161/01.STR.0000217222.09978.ce

24. Ruland S, Raman R, Chaturvedi S, Leurgans S, Gorelick PB, Study AIAAASP. Awareness, treatment, and control of vascular risk factors in African Americans with stroke. Neurology. 2003;60(1):64-68. doi:10.1212/WNL.60.1.64

25. Christian JB, Lapane KL, Toppa RS. Racial disparities in receipt of secondary stroke prevention agents among US nursing home residents. Stroke J Cerebral Circulat. 2003;34(11):2693-2697. doi:10.1161/01.STR.0000096993.90248.27

26. Tuhrim S. Editorial comment-ethnic disparities in stroke: epidemiology, acute care, and postacute outcomes. Stroke J Cerebral Circulat. 2005;36(2):386-387. doi:10.1161/01.STR.0000153060.06006.00

27. Perzynski A, Blixen C, Cage J, Colon-Zimmermann K, Sajatovic M. Informing policy for reducing stroke health disparities from the experience of African-American male stroke survivors. J Racial Ethn Health Disparities. 2016;3(3):527-536. doi:10.1007/s40615015-0171-2

28. Stansbury JP, Jia H, Williams LS, Vogel WB, Duncan PW. Ethnic disparities in stroke: epidemiology, acute care, and postacute outcomes. Stroke J Cerebral Circulat. 2005;36(2):374-386. doi:10.1161/01.STR.0000153065.39325.fd

29. Helgason CM, Wolf PA. American Heart Association prevention conference IV: prevention and rehabilitation of stroke: executive summary. Circulation. 1997;96(2):701-707. doi:10.1161/01. CIR.96.2.701

30. Foster G, Taylor SJ, Eldridge SE, Ramsay J, Griffiths CJ. Selfmanagement education programmes by lay leaders for people with chronic conditions. Cochrane Database Syst Rev. 2007;4:Cd005108.

31. Lennon S, McKenna S, Jones F. Self-management programmes for people post stroke: a systematic review. Clin Rehabil. 2013;27 (10):867-878. doi:10.1177/0269215513481045

32. Battersby M, Hoffmann S, Cadilhac D, Osborne R, Lalor E, Lindley R. 'Getting your life back on track after stroke: a phase II multi-centered, single-blind, randomized, controlled trial of the stroke self-management program vs. the stanford chronic condition self-management program or standard care in stroke survivors. Int J Stroke. 2009;4(2):137-144. doi:10.1111/ j.1747-4949.2009.00261.x

33. Goldfinger JZ, Kronish IM, Fei K, et al. Peer education for secondary stroke prevention in inner-city minorities: design and methods of the prevent recurrence of all inner-city strokes through education randomized controlled trial. Contemp Clin Trials. 2012;33(5):1065-1073. doi:10.1016/j.cct.2012.06.003

34. Kronish IM, Goldfinger JZ, Negron R, et al. Effect of peer education on stroke prevention: the prevent recurrence of all inner-city strokes through education randomized controlled trial. Stroke. 2014;45 (11):3330-3336. doi:10.1161/STROKEAHA.114.006623 
35. Plow M, Moore SM, Sajatovic M, Katzan I. A mixed methods study of multiple health behaviors among individuals with stroke. PeerJ. 2017;5:e3210. doi:10.7717/peerj.3210

36. Sajatovic M, Tatsuoka C, Welter E, et al. A targeted self-management approach for reducing stroke risk factors in African American men who have had a stroke or transient ischemic attack. Am J Health Promot. 2018;32(2):282-293. doi:10.1177/0890117117695218

37. Ryan P, Sawin KJ. The individual and family self-management theory: background and perspectives on context, process, and outcomes. Nurs Outlook. 2009;57(4):217-225 e216. doi:10.1016/j. outlook.2008.10.004

38. Johnston SC, Rothwell PM, Nguyen-Huynh MN, et al. Validation and refinement of scores to predict very early stroke risk after transient ischaemic attack. Lancet. 2007;369(9558):283-292. doi:10.1016/ S0140-6736(07)60150-0

39. Josephson SA, Sidney S, Pham TN, Bernstein AL, Johnston SC. Higher ABCD2 score predicts patients most likely to have true transient ischemic attack. Stroke J Cerebral Circulat. 2008;39 (11):3096-3098. doi:10.1161/STROKEAHA.108.514562

40. Rothwell PM, Giles MF, Flossmann E, et al. A simple score (ABCD) to identify individuals at high early risk of stroke after transient ischaemic attack. Lancet. 2005;366(9479):29-36. doi:10.1016/ S0140-6736(05)66702-5

41. Kasner SE. Clinical interpretation and use of stroke scales. Lancet Neurol. 2006;5(7):603-612. doi:10.1016/S1474-4422(06)70495-1

42. Mahoney FI, Barthel DW. Functional evaluation: the Barthel index. Md State Med J. 1965;14:61-65.

43. U.S. Census Bureau. U.S. census Bureau quick facts. 2018. Available from: https://www.census.gov/quickfacts/fact/table/cuyahogacountyo hio,OH/PST045217. Accessed October 1, 2020.

44. Sajatovic M, Gunzler DD, Kanuch SW, et al. A 60-week prospective RCT of a self-management intervention for individuals with serious mental illness and diabetes mellitus. Psychiatr Serv. 2017;68 (9):883-890. doi:10.1176/appi.ps.201600377

45. Chobanian AV, Bakris GL, Black HR, et al. The seventh report of the Joint National Committee on prevention, detection, evaluation, and treatment of high blood pressure: the JNC 7 report. JAMA. 2003;289 (19):2560-2572. doi:10.1001/jama.289.19.2560

46. Johnson KC, Whelton PK, Cushman WC, et al. Blood pressure measurement in SPRINT (systolic blood pressure intervention trial). Hypertension. 2018;71(5):848-857. doi:10.1161/HYPERTENSI ONAHA.117.10479

47. Cohen S, Kamarck T, Mermelstein R. A global measure of perceived stress. J Health Soc Behav. 1983;24(4):385-396. doi:10.2307/ 2136404

48. Cohen S, Williamson G. Perceived stress in a probability sample of the United States. In: Spacapam S, Oskamp S, editors. The Social Psychology of Health: Claremont Symposium on Applied Social Psychology. Newbury Park, CA: Sage; 1988.

49. Cella D, Riley W, Stone A, et al. The patient-reported outcomes measurement information system (PROMIS) developed and tested its first wave of adult self-reported health outcome item banks: 2005-2008. J Clin Epidemiol. 2010;63(11):1179-1194. doi:10.1016/ j.jclinepi.2010.04.011

50. Cella D, Yount S, Rothrock N, et al. The patient-reported outcomes measurement information system (PROMIS): progress of an NIH Roadmap cooperative group during its first two years. Med Care. 2007;45(5 Suppl 1):S3-S11. doi:10.1097/01. mlr.0000258615.42478.55

51. Williams DR, Yan Y, Jackson JS, Anderson NB. Racial differences in physical and mental health: socio-economic status, stress and discrimination. J Health Psychol. 1997;2(3):335-351. doi:10.1177/ 135910539700200305
52. Dolezsar CM, McGrath JJ, Herzig AJ, Miller SB. Perceived racial discrimination and hypertension: a comprehensive systematic review. Health Psychol. 2014;33(1):20-34. doi:10.1037/a0033718

53. Arozullah AM, Yarnold PR, Bennett CL, et al. Development and validation of a short-form, rapid estimate of adult literacy in medicine. Med Care. 2007;45(11):1026-1033. doi:10.1097/ MLR.0b013e3180616c1b

54. Mistry R, Gokhman I, Bastani R, et al. Measuring medical burden using CIRS in older veterans enrolled in UPBEAT, a psychogeriatric treatment program: a pilot study. J Gerontol a Biol Sci Med Sci. 2004;59(10):1068-1075. doi:10.1093/gerona/59.10.M1068

55. Pilkerton CS, Singh SS, Bias TK, Frisbee SJ. Changes in cardiovascular health in the United States, 2003-2011. J Am Heart Assoc. 2015;4(9):e001650. doi:10.1161/JAHA.114.001650

56. Pucciarelli G, Buck HG, Barbaranelli C, et al. Psychometric characteristics of the mutuality scale in stroke patients and caregivers. Gerontologist. 2016;56(5):e89-98. doi:10.1093/geront/gnw083

57. Sullivan K, Dunton NJ. Development and validation of the stroke knowledge test. Top Stroke Rehabil. 2004;11(3):19-28. doi:10.1310/ RED5-V47T-8MJN-JY9H

58. Ellis C. An examination of stroke-related knowledge in male stroke survivors living in the South Carolina Stroke Belt. Am J Mens Health. 2014;8(6):532-537. doi:10.1177/1557988314531038

59. Schwartzer R, Jerusalem M. Generalized self-efficacy scale. In: Measures in Health Psychology: A User's Portfolio, Causal and Control Beliefs. Windsor, England: NFER-Nelson; 1995:35-37.

60. Vauth R, Kleim B, Wirtz M, Corrigan PW. Self-efficacy and empowerment as outcomes of self-stigmatizing and coping in schizophrenia. Psychiatry Res. 2007;150(1):71-80. doi:10.1016/j. psychres.2006.07.005

61. Sherbourne CD, Stewart AL. The MOS social support survey. Soc Sci Med. 1991;32(6):705-714. doi:10.1016/0277-9536(91)90150-B

62. Hays RD, Sherbourne CD, Mazel RM. User's Manual for Medical Outcomes Study (MOS) Core Measures of Health-Related Quality of Life. RAND Corporation: Santa Monica, CA; 1995.

63. Conway JM, Ingwersen LA, Moshfegh AJ. Accuracy of dietary recall using the USDA five-step multiple-pass method in men: an observational validation study. J Am Diet Assoc. 2004;104(4):595-603. doi:10.1016/j.jada.2004.01.007

64. Tran KM, Johnson RK, Soultanakis RP, Matthews DE. In-person vs telephone-administered multiple-pass 24-hour recalls in women: validation with doubly labeled water. $J$ Am Diet Assoc. 2000;100 (7):777-783. doi:10.1016/S0002-8223(00)00227-3

65. Rubenstein JH, Morgenstern H, Kellenberg J, et al. Validation of a new physical activity questionnaire for a sedentary population. Dig Dis Sci. 2011;56(9):2678-2687. doi:10.1007/s10620-011-1641-y

66. Fagerström KO. Measuring degree of physical dependence to tobacco smoking with reference to individualization of treatment. Addict Behav. 1978;3(3-4):235-241. doi:10.1016/0306-4603(78)90024-2.

67. Doolan DM, Froelicher ES. Smoking cessation interventions and older adults. Prog Cardiovasc Nurs. 2008;23(3):119-127. doi:10.1111/j.1751-7117.2008.00001.x

68. Mdege ND, Lang J. Screening instruments for detecting illicit drug use/abuse that could be useful in general hospital wards: a systematic review. Addict Behav. 2011;36(12):1111-1119. doi:10.1016/j. addbeh.2011.07.007

69. Resource guide: screening for drug use in general medical settings. National Institute on Drug Abuse; Available from: https://archives. drugabuse.gov/publications/resource-guide-screening-drug-use-ingeneral-medical-settings. Accessed September 8, 2020. 


\section{Publish your work in this journal}

The Journal of Multidisciplinary Healthcare is an international, peerreviewed open-access journal that aims to represent and publish research in healthcare areas delivered by practitioners of different disciplines. This includes studies and reviews conducted by multidisciplinary teams as well as research which evaluates the results or conduct of such teams or healthcare processes in general. The journal covers a very wide range of areas and welcomes submissions from practitioners at all levels, from all over the world. The manuscript management system is completely online and includes a very quick and fair peer-review system. Visit http://www.dovepress.com/testimonials. php to read real quotes from published authors. 